EOMmUn Communication et organisation

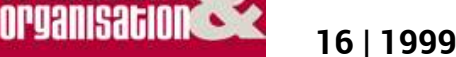

Crise et communication

\title{
Crise du mécénat ou mécénat de crise?
}

\section{Nicole Denoit}

\section{OpenEdition}

Journals

Édition électronique

URL : http://journals.openedition.org/communicationorganisation/2260

DOI : 10.4000/communicationorganisation.2260

ISSN : 1775-3546

\section{Éditeur}

Presses universitaires de Bordeaux

\section{Édition imprimée}

Date de publication : 1 novembre 1999

ISSN : 1168-5549

\section{Référence électronique}

Nicole Denoit, "Crise du mécénat ou mécénat de crise ? », Communication et organisation [En ligne]

16 | 1999, mis en ligne le 27 mars 2012, consulté le 19 avril 2019. URL : http://

journals.openedition.org/communicationorganisation/2260; DOI : 10.4000/

communicationorganisation.2260

Ce document a été généré automatiquement le 19 avril 2019.

(c) Presses universitaires de Bordeaux 


\title{
Crise du mécénat ou mécénat de crise?
}

\author{
Nicole Denoit
}

1 Parler de communication de crise, c'est d'abord devoir constater la difficulté à définir le mot crise qui «s'est répandu de proche en proche envahissant toute chose sociale, toute notion $»^{1}$. Perdant tout contour, la notion de crise "permet tout au plus de dire que quelque chose ne va pas $»^{2}$.

2 De quelle crise parle-t-on ici ? De la crise économique qui débute dès 1973 avec des rémissions et des poussées, de la crise des valeurs et de la dégradation du lien social, de la crise qui affecte spécifiquement certaines entreprises mécènes dans la tourmente des scandales financiers? On retiendra la complexité de la notion de crise.

3 Parle-t-on de ce qu'on peut appeler une crise du mécénat d'entreprise dans les années 92-93, soit un recul des investissements, ou d'un mécénat de crise, c'est-à-dire d'une forme nouvelle de mécénat née de la crise?

4 S'intéresser à la « communication de crise » ce peut être, en effet, considérer les effets de la crise sur la communication, donc cerner la notion à travers ses conséquences sur un champ spécifique, celui de la communication, plus précisément sur le terrain du mécénat. On peut donc attendre de cette étude, à la fois une approche renouvelée de la notion de "crise », par l'angle d'observation spécifique qu'est le mécénat dans le champ de la communication d'entreprise, mais aussi, par une analyse des effets de la crise sur les actions de mécénat, une meilleure compréhension du fonctionnement de l'organisation dans ses relations avec l'environnement.

5 L'étude de symptômes précis devrait contribuer à une meilleure connaissance de la communication des entreprises par le mécénat mais aussi à une «crisologie » dans le sens que lui donne Édgar Morin. Parce que « la crise est à la fois un révélateur et un effecteur » elle « révèle ce qui était caché, latent, virtuel au sein de la société [...] » et en même temps elle «nous éclaire théoriquement sur la part immergée de l'organisation sociale, sur ses capacités de survie et de transformation $»^{3}$.

6 Nous confirmons par notre étude que la crise révèle, par son incidence sur le mécénat, des traits importants de la stratégie de communication des entreprises dans la période 
faste des années 80 : derrière la démarche consensuelle du mécénat culturel, encouragée par le «tout culturel» de la politique gouvernementale, l'entreprise dissimule son inaptitude à intégrer les éléments extérieurs, notamment ceux de la crise économique.

7 Cette inaptitude nous semble correspondre à ce que d'autres analyses désignent dans le fonctionnement d'une entreprise "instituante ${ }^{4}$, c'est-à-dire normative, directive. Il s'agirait moins de réagir à l'environnement, de s'y adapter que de le modeler.

8 Si le mécénat culturel des années 80 apparaît consensuel, en raison de l'idéalisation à la fois de l'entreprise et de l'art, il reste une vitrine, voire un écran qui dissimule non seulement la réalité de l'entreprise mais la réalité à l'entreprise. L'entreprise, pour se faire " instituante ${ }^{5}$, simule une position de sujet qu'elle confisque aux véritables sujets, en prétendant à une identité à laquelle il faut adhérer, qu'il faut assimiler.

9 Les scandales financiers de certaines grandes entreprises mécènes confirment le décalage du mécénat d'entreprise des années 80 , élégante et généreuse vitrine sociale, par rapport à la réalité de l'entreprise de cette période, déjà jetée loin du mécénat dans la jungle de la concurrence et du profit, souvent personnel.

10 Apparaît alors une période de dégradation, de crise du mécénat dont nous prendrons la mesure. La crise joue ensuite, après l'épreuve des années 1992-93, son rôle d'« effecteur ». Elle aura permis à l'entreprise de mieux comprendre « la part immergée de l'organisation sociale » et, en elle-même, ses « capacités de survie et de transformation » qui donneront naissance à un mécénat nouveau, non plus de solidarité mais réellement solidaire, dans une compréhension des phénomènes d'interaction sociale. Qu'elle soit culturelle ou sociale, cette solidarité dite "citoyenne" débouche sur une responsabilisation, une individualisation et une autonomisation du salarié désormais impliqué, à titre personnel, dans une mission de mécénat qu'il initie et que l'entreprise soutient.

11 Notre étude procédera en trois temps. Nous observerons le décalage entre la politique de mécénat des années 80 et la crise économique pour mieux comprendre la crise du mécénat des années 92-93 qui donnera naissance à une nouvelle stratégie de communication, celle d'une entreprise consciente de l'interdépendance sociale et de ce fait réellement solidaire de son environnement.

12 En d'autres termes, que nous reprenons à l'analyse de Nicole d'Alméida, nous pouvons observer le passage de l'entreprise «instituante des années 80 , directive, visant à faire intérioriser les normes, les valeurs et, pour ce qui nous concerne, une entreprise mécène certes, mais directive, à l'entreprise des années 90, « instituée », c'est-à-dire intégrant les diverses contraintes de son environnement ». Cette entreprise "instituée », aux normes intériorisées, serait-elle une entreprise non seulement solidaire, interdépendante, mais " civilisée », au sens où Norbert Élias entend ce mot, c'est-à-dire précisément lorsque les normes, les contraintes sociales sont assez intériorisées pour permettre aux individus une autodiscipline?

\section{Le mécénat d'entreprise des années 80 : une élégante vitrine « citoyenne »}

\section{L'entreprise séduite}

13 Médiatisée à l'extrême, représentée dans l'opinion publique par quelques ténors au parler généreux et populaire, l'entreprise s'est prise au jeu de la citoyenneté. Elle cristallise les 
espérances à défaut des autres secteurs pour le moins en déficit d'image : religion, politique...

L'entreprise est séduite par sa propre image et le mécénat des années 80 s'apparente ici aux «conduites désintéressées qui n'ont pas pour principe le calcul de désintéressement $»^{6}$. En reprenant l'analyse de Pierre Bourdieu à propos des sociétés d'honneur et des habitus désintéressés, on peut dire que l'entreprise, comme l'aristocrate, idéalisée par le corps social, ne peut faire autrement que d'être généreuse par fidélité à un groupe et par fidélité à elle-même comme digne d'être membre du groupe.

Le mécénat d'entreprise s'apparente ici aux « conduites désintéressées qui n'ont pas pour principe le calcul de désintéressement ${ }^{7}$. En reprenant l'analyse de Pierre Bourdieu à propos des sociétés d'honneur et des habitus désintéressés, on peut dire que l'entreprise, comme l'aristocrate, idéalisée par le corps social, ne peut faire autrement que d'être généreuse par fidélité à un groupe et par fidélité à elle-même comme digne d'être membre du groupe.

Si l'entreprise cristallise les espérances sociales, l'art comble le vide spirituel laissé par la religion et la politique. Les années 80 , les "années fric ", selon l'expression d'AlainDominique Perrin, Président de la Fondation Cartier, mettent en place un mécénat culturel de prestige, élégante vitrine pour la communication externe, en forçant le trait, ce qu'on a pu appeler un « mécénat petits-fours».

17 Certes, dans les années 80 , «les rituels de relations publiques se seraient socialisés, banalisés et répandus $»^{8}$ et leurs représentations pourraient même servir aux publicités institutionnelles.

Si nous retenons cette idée de «l'extension de ce qui peut être considéré comme la sphère interne de l'entreprise ${ }^{9}$, il n'en reste pas moins que le mécénat est conçu par les dirigeants de l'entreprise comme un élément de « distinction » exportable.

La fascination pour l'entreprise, sa médiatisation, la font entrer dans le star system, dans une société du spectacle et du paraître dont s'inquiète en 1988 Claude Marti, président d'honneur de la Fédération de la publicité française, conseiller en communication de François Mitterrand : «Depuis quatre ans, la société française s'est mise à vivre en pleine artificialité et cela m'inquiète. On peut coloniser tout un pays par la séduction $»^{10}$.

20 L'entreprise des années 80 est séduite: par sa propre image d'abord, sous l'influence d'une opinion prête à croire à la citoyenneté d'une entreprise dont on attend tous les bienfaits, mais aussi par un "État séducteur", pourrions-nous dire dans un léger détournement de la formule de Régis Debray.

21 Croire que la culture pouvait être l'affaire de tous présentait l'avantage pour l'État d'un soulagement financier et peut-être d'une diversion politique. On a pu dire en effet que « la gauche au pouvoir aura dépolitisé la politique » et qu'«à la place elle mit au premier plan l'économie, mais il est difficile de mobiliser le peuple de gauche sur la désinflation compétitive. Il lui fallait quelques valeurs : la communication et la culture. Peu à peu, elle déplaça son identité du champ social au champ culturel. Pour elle la culture ne fut pas un ornement, ni un miroir aux alouettes, mais un instrument essentiel, plus idéologique que matériel, certes, mais qui permit de rallier largement [...]. La culture fut le substitut d'une politique absente $»^{11}$.

22 L'État officialise le mécénat d'entreprise et la loi de 1987 accorde des avantages fiscaux aux entreprises mécènes qui agissent sans recherche de contrepartie et en vue seulement 
de l'intérêt général. Certes, peu d'entreprises soutiendront le paradoxe, pour elles, d'une action désintéressée et, reconnaissant l'attente d'un retour d'image, préféreront médiatiser leurs actions et déduire leurs frais de mécénat en frais de publicité. L'entreprise fut cependant incitée au mécénat par son officialisation qui en fait un acte normal de gestion reconnu et honoré par l'État.

L'entreprise fut-elle aspirée par cette substitution de la culture à la politique, son discours de citoyenneté est-il aussi un évitement des problèmes que la crise économique fera apparaître en son sein?

Le mécénat culturel est consensuel et en ce sens il est fédérateur et repousse pour un temps les antagonismes latents, «les forces d'opposition et de dissociation, c'est-à-dire les tendances à la dispersion $»^{12}$ présentes dans toute organisation. Or, selon les termes de la «crisologie » d'Édgar Morin, plus un système est clos, moins il est à l'abri de la désintégration et de la dispersion parce qu'il ne peut se restaurer en puisant de l'énergie et de l'organisation à l'extérieur $»^{13}$.

Le mécénat des années 80, paradoxalement, puisque la notion de mécénat implique une générosité tournée vers l'extérieur, vers l'Autre, ne fonctionne pas en système véritablement ouvert. Il manifeste la cristallisation des espérances sociales sur l'entreprise, la vulnérabilité également de l'entreprise à la séduction d'un État culturel plutôt que politique, entreprise qui n'intègre pas la crise économique et sociale déjà engagée, qui ne se remet pas en question.

Le mécénat de l'UAP nous semble un exemple significatif d'une stratégie d'entreprise soumise au pouvoir de l'État et inconsciente du décalage dangereux avec les réalités économiques.

Le décalage est déjà repérable au niveau des dates: en 1991, lorsque l'UAP engage son soutien à la Galerie Nationale du Jeu de Paume, le mécénat culturel de beaucoup d'entreprises est en repli, du moins en transformation, dans la conscience qu'une stratégie sociale s'impose, qu'un mécénat de solidarité, qui n'exclut pas le secteur culturel à condition qu'il soit abordé comme secteur d'insertion, doit être privilégié. Le mécénat culturel continue mais plus discrètement.

Dès 1986, Claude Bébéar prend l'initiative de créer l'Institut du mécénat humanitaire et initie chez AXA un mécénat de solidarité qui implique les salariés de l'entreprise et se montre à l'écoute des problèmes sociaux qui les mobilisent. Par contre, en 1992 encore, l'UAP est fière d'articuler, sur les hardiesses d'un art contemporain difficile d'accès, une campagne de publicité provocatrice. Ainsi ce slogan «On est numéro un oui ou non?", suivi d'une campagne d'affichage grand public qui accompagne, dans le même esprit impertinent, une œuvre difficile d'Hélio Ottica: «Il serait temps d'avoir une bonne explication avec l'art contemporain ».

La politique de communication par le mécénat, dans les années 80, est narcissique, « raffinée », selon les termes de Jacques Rigaud, Président d'Admical (association pour le développement du mécénat industriel et commercial, créée en 1979 pour promouvoir le mécénat culturel, et qui ne s'ouvrira qu'en 1992, contrainte par l'évolution devenue évidente, au mécénat de solidarité). Elle est sous le charme aussi d'une politique de séduction de l'État qui y trouve son compte pendant un temps. Du prince au président, l'esprit du mécénat culturel des années 80 reste celui d'une politique de prestige, certes au service des arts, mais moins du public, et en décalage avec la crise économique et sociale qui s'installe durablement. 


\section{L'entreprise décrédibilisée} " Prince ", lié au pouvoir et à ses fluctuations et on confirmera sur le terrain du mécénat que ces acteurs au pouvoir «peuvent avoir des conduites raisonnables sans être rationnels", qu'«ils peuvent avoir des conduites dont on peut rendre raison, comme disaient les classiques, à partir de l'hypothèse de la rationalité, sans que ces conduites aient eu la raison pour principe $\aleph^{14}$, même si les apparences et les discours veulent le faire croire. rationnels, mais révélateurs de ce qui reste toujours d'engagement personnel et 
passionnel dans le mécénat, entraînant des réactions épidermiques qui coûtent parfois cher au mécénat et à l'entreprise.

Ainsi la dissolution de la Fondation Elf, le 17 décembre 1996, par la décision brutale Philippe Jaffré, Président-Directeur général, prive l'entreprise des 36 millions de francs de la Fondation qui, selon les statuts, passent à la Croix Rouge.

La crise qui affecte le mécénat d'Elf aquitaine apparaît directement liée à la mise en examen et à l'incarcération de son ancien dirigeant Loïc Le Floch Prigent. Partisan convaincu du mécénat d'entreprise, après avoir créé la Fondation Elf, il se montre précurseur d'une forme de mécénat qui implique en interne les salariés : le «mécénat associé ». Dès son arrivée à la direction de la SNCF, il crée la Fondation Solidarité SNCF.

Nul doute que la décision de Philippe Jaffré de faire disparaitre la Fondation a suscité surprise, doutes et rumeurs.

41 Comment l'expliquer? Deux raisons sont avancées par le groupe : le souhait de chercher "un mode de fonctionnement plus en accord avec la politique de communication de l'entreprise", mais principalement le souci de briser le dernier lien entre l'entreprise et la tutelle publique (le conseil d'administration d'une fondation reconnue d'utilité publique accueillant, en effet, des représentants de l'État) ${ }^{15}$. On comprend encore mieux ce désir depuis les révélations de l'«affaire Dumas». Les moyens de séduction de l'État sont souvent irrésistibles pour l'entreprise et ceux qui la dirigent mais réciproquement.

Sur le terrain de la séduction, il n'est pas question pour l'entreprise d'intégrer des contraintes, de s'adapter, mais de céder souvent aveuglément et dangereusement à la tentation. Ainsi, l'évolution de l'entreprise « instituante » des années 80 vers l'entreprise « instituée » des années 90, rencontre entre ces deux étapes, l'entreprise « séduite ».

\section{Des engagements contestés}

43 Le mécénat humanitaire des années 80 , consensuel, inévitable même - qui ne lui apporterait pas son soutien passerait pour insensible et condamnable ${ }^{16}$ - et qui est donc largement encouragé par les entreprises, va être reconsidéré dans son efficacité par les entreprises mécènes qui s'aperçoivent qu'elles obtiennent par leur engagement un effet souvent inverse à celui qui était attendu, non seulement auprès des bénéficiaires mais auprès des salariés et de l'opinion.

En effet, du côté des prétendus bénéficiaires du mécénat, les aides sont détournées au profit des luttes politiques qu'elles contribuent à prolonger sur le terrain, tandis que l'engagement humanitaire, «idéologie de secours $»^{17}$, évite aux politiques de prendre les décisions nécessaires. Prise de conscience donc d'un détournement du mécénat humanitaire à tous les niveaux.

Le 28 octobre 98, on lisait encore dans le journal Libération sous le titre «Sud-Soudan, cimetière de l'humanitaire » que « l'aide internationale n'a pas arrêté la famine de cet été. Détournée, elle a d'abord profité aux chefs de guerre [...] Les organisations humanitaires, qui avaient roulé le tambour de la compassion, n'ont aucun intérêt à une évaluation indépendante de leur action au Soudan. Hésitante au début, elle a fini par être techniquement impressionnante, tout en produisant de piètres résultats [...] L'opération d'aide a été prise en otage par les maquisards soudanais [...] Les détournements ne gênent pas les principaux bailleurs de fonds, à commencer par les Etats-Unis, tant qu'ils se font au détriment du régime islamiste de Khartoum ». L'entreprise peut donc avoir à se 
justifier de son manque de lucidité face au cynisme des politiques dont elle fait le jeu, innocemment ou stratégiquement.

Certes, il s'agit là du point de vue des médias mais une approche théorique du mécénat ne peut négliger le fait que ce choix de communication par le mécénat est engagé en direction d'une opinion soumise à l'influence des médias, et que, par ailleurs, les entreprises le savent, l'efficacité de cette politique de communication nécessite le soutien médiatique aux actions de l'entreprise mécène.

Les acteurs eux-mêmes remettent en question l'enjeu humanitaire. On pense bien sûr à Rony Brauman, Président de Médecins sans frontières de 1982 à 1994, qui dénonce en 1996 dans Les médias et l'humanitaire, la « charité-spectacle », « la charity-business », ou encore la " pitié dangereuse ».

Le mécénat humanitaire des entreprises est non seulement confronté aux erreurs, aux hypocrisies de cette "idéologie de secours" partout dénoncées, mais aussi à la malhonnêteté qu'un rapport de la commission européenne révèle à propos de « l'absence de contrôle des dépenses humanitaires » et des 6,4 milliards de francs français qui ont été dépensés «sans disposer d'aucune facture et sans aucune garantie quant à la réalité des chiffres qui lui sont remis $»^{18}$.

Malhonnêteté de sociétés françaises, complicité d'un fonctionnaire de la commission : il est difficile pour les entreprises mécènes de communiquer désormais sans risque sur leur politique de soutien à l'humanitaire. Si elles maintiennent leurs programmes, c'est réellement par conviction et dans une discrétion qui nous éloigne du "protocole compassionnel $»^{19}$, culminant en Somalie.

Les entreprises ont cédé dans les années 80 à la rhétorique vertueuse des "Shows philanthropiques ${ }^{20}$ qui avait saisi tous les esprits et déculpabilisait tout un chacun à bon compte.

51 Ainsi, la crise joue un rôle de révélateur de ce qui était latent dans la société et des ressources d'adaptabilité et d'intégration dont l'entreprise doit et peut faire preuve.

Comme le souligne E. Morin, plus le système est clos, moins il est à l'abri de la désintégration et de la dispersion parce qu'il ne peut se restaurer en puisant de l'énergie et de l'organisation à l'extérieur [...], la seule possibilité de lutter contre la désintégration est d'intégrer et d'utiliser le plus possible les antagonismes de façon organisationnelle; de renouveler énergie et organisation en les puisant dans l'environnement (système ouvert) ${ }^{21}$.

53 Le mécénat, dans le cas où il n'est pas seulement le miroir dans lequel l'entreprise narcissique des années 80 se trouve encore plus belle que l'opinion ne le pense, est logiquement une ouverture sur l'environnement qui va préparer l'entreprise à intégrer la crise, à s'adapter, tout en faisant évoluer son mécénat de solidarité vers un mécénat solidaire qui explicite cette adaptabilité.

Cependant, gardons-nous à propos du mécénat d'une "vision enchantée ", pour nous apercevoir que cette adaptation nécessaire est une réponse de l'entreprise aux contraintes de plus en plus pressantes de son environnement et l'on affirmerait abusivement que c'est délibérément qu'elle passe du stade d'entreprise « instituante » au stade d'entreprise « instituée ». 


\section{Le mécénat d'entreprise en temps de crise : une mise en évidence de l'interdépendance sociale}

Le chômage fait davantage d'exclus et l'entreprise, comme chaque citoyen concerné par la crise - dont désormais personne ne se sent protégé - veut agir au plus près, au plus vite et au plus efficace et veut pouvoir apprécier les réels besoins tout en mesurant les progrès liés à l'aide apportée. L'entreprise va articuler son action de mécénat sur les initiatives locales, notamment celles des associations et en particulier celles dans lesquelles ses salariés sont engagés à titre individuel.

56 Le développement du mécénat de solidarité est parallèle à une implication du personnel et à une transparence des politiques engagées.

\section{Le développement du mécénat de solidarité dans les années 90}

57 Dans le Cahier du mécénat solidarité de La Lettre du Mécénat de novembre 92 un entretien avec René Barberye, Président du Directoire du Centre National des Caisses d'Épargne et de Prévoyance fait état d'une nouvelle orientation du mécénat : les Caisses d'Épargne se sont engagées à doubler les sommes provenant du Fonds Commun de Placement. Écureuil Recherche et Partage a été lancé en 91. «Il est vrai que ce qu'on appelle l'argent Éthique est un concept nouveau en France mais je suis convaincu qu'il s'affirmera progressivement. J'ai bon espoir que Recherche et Partage y concourra efficacement » déclare René Barberye.

En 93, dans le même Cahier du mécénat solidarité, Jacques Rigaud, Président d'Admical précise : « Lorsque nous lancions ce cahier du mécénat solidarité pour nous faire l'écho de la diversification des thèmes auxquels s'intéressaient les adhérents de l'Admical nous ne pensions pas que l'évolution serait aussi rapide [...]. Le mécénat de solidarité semble devenu de droit commun. Dans le même temps, ses formes, ses interventions se diversifient : partenariats complexes avec les bénéficiaires, opérations pluri-entreprises, créations de véritables services sociaux (Nestlé), initiative de plus en plus affirmée du personnel et apports de compétences pointues (Elf Aquitaine). S'agit-il encore de mécénat dans l'acceptation habituelle du terme?

59 Les frontières s'estompent avec d'autres fonctions de l'entreprise: gestion sociale, marketing, formation... Nous n'entrerons pas ici dans ce débat. Nous nous bornerons à constater que les entreprises s'engagent de façon plus résolue dans des activités d'intérêt général qui ne sont pas directement nécessaires à la réalisation de leur objet social.

On perçoit bien la réserve de Jacques Rigaud qui a toujours œuvré pour le développement du soutien aux arts, à ce qu'il nomme «mécénat culturel », mécénat dont la générosité était évaluée à la distance de l'engagement mécène d'avec l'objet social de l'entreprise.

61 Le mécénat se déploie désormais dans une interdépendance sociale complexe car elle nécessite de nouveaux moyens d'identification de ce qui relève du mécénat ou de la réactivité nécessaire de l'organisation à son environnement. La démarche de mécénat est autre chose qu'une adaptation de l'entreprise aux nouvelles contraintes de son environnement. 


\section{L'articulation du social et du culturel : le « mécénat croisé "} Paume, de la Fondation Cartier et de son soutien à l'art contemporain - il était le fait des dirigeants d'entreprise parfois formés à la culture classique mais souvent le fait de dirigeants d'entreprises avides d'asseoir leur pouvoir économique sur une reconnaissance culturelle en entrant dans le cénacle de ceux qui approchent la culture d'avant-garde officialisée par l'État.

«Dans ce temps d'officialisation de l'avant-garde, on a assisté au cours des dix dernières années à une extension spectaculaire du champ des interventions culturelles de l'État [...] Extension par contiguïté de la musique au rap, de la fresque au tag, de la littérature à la bande dessinée. Extension par bonds : la couture, l'art équestre, la cuisine, l'art forain. Grâce au ministère de la Culture, il y a désormais des arts de la table... ${ }^{22}$.

«En fait, le ministère de la Culture tantôt dit «tout est art, tout le monde est artiste ", tantôt contradictoirement que seul le moderne d'avant-garde est artiste. Il est vrai, la politique n'est pas tenue à la logique et peut fort bien louer également, quoique subventionner inégalement, le rap (il ne faut pas désespérer la Courneuve) et le sérialisme généralisé (il ne faut pas désespérer le Lubéron) $»^{23}$. croisé » qui associe le culturel et le social. La crise souligne la confusion dans les années 80 de la culture et du culturel et le mécénat des années 90 tire le meilleur parti de cette confusion, à l'heure où les problèmes sociaux à résoudre apparaissent comme une priorité aux entreprises et aux salariés. La montée du culturel aura préparé un mécénat d'intégration solidaire qui cherche des solutions sociales du côté du culturel et non de la culture qui n'a pas vocation, elle, d'intégrer. 


\section{La crise des années 92-93 : vers un mécénat d'initiative}

70 L'évolution du mécénat dans les années 92-93 a révélé les contradictions de la politique culturelle des années 80 et a tiré le meilleur parti des conséquences de cette politique. Certains, comme J. Rigaud déplorent discrètement, parce qu'ils restent attachés à la définition du mécénat comme soutien à l'art, le glissement de la culture au culturel, puis du culturel au social.

71 À partir de 1992, culture et solidarité sont associées dans les actions de mécénat qui font le plus parler d'elles: Nicolas Frize et le concert qu'il organise avec des détenus aux longues peines, Armand Gatti et son travail de théâtre avec des jeunes marginalisés... Les entreprises se passionnent pour ces démarches associées. Admical s'inquiète: «La vigilance est de mise : une œuvre d'art, quelle qu'elle soit, ne saurait se réduire à son utilité sociale, réelle ou supposée, et il serait périlleux pour la création artistique que les pouvoirs publics ou les mécènes privés ne se déterminent qu'en fonction de ce seul critère $»^{24}$.

Les entreprises ont pris l'initiative du mécénat de solidarité et vont bientôt laisser cette initiative à leurs salariés pour qu'ils s'engagent dans un mécénat solidaire, une action de partage des responsabilités sociales.

73 La fondation Elf montre le chemin et s'oriente vers un mécénat de compétences qui implique le personnel. Ainsi à propos de deux opérations d'envergure, l'université d'Alexandrie et le Galion à San Diego «Elle veut de plus en plus impliquer ses salariés dans ses actions. Outre l'aide technique et intellectuelle qu'apporte le personnel d'encadrement aux projets, les salariés sont invités à proposer des projets ou à verser des dons à des associations choisies collectivement $»^{25}$.

74 En 93 l'engagement des entreprises dans la lutte contre l'exclusion va se confirmer. La crise prend des formes sociales très visibles : « chômage, exclusion, drogue, racket, haine raciale, marginalisation, échec scolaire, alcoolisme, délinquance, dégradation de l'habitat... tous ces termes ont le triste privilège d'être évoqués lorsqu'on fait référence aux quartiers urbains défavorisés » lit-on dans un «État des lieux » proposé par La Lettre du mécénat de février-mars 1993. La mobilisation est urgente : 3 millions de personnes concernées, 500 quartiers retenus par l'État pour faire l'objet d'une convention entre les communes, l'État et les régions. Les énergies se mobilisent : individuelles, associatives...

« Les entreprises tentent de plus en plus d'apporter des solutions qui, pour minimes qu'elles soient, prouvent qu'une action organisée et bien gérée peut endiguer les phénomènes de marginalité et d'exclusion ${ }^{26}$.

C'est l'heure des actions communes entre entreprises et collectivités locales. Les Assises du mécénat de 96 (après celles de 94 intitulées "Épreuves et preuves du mécénat ») s'annoncent sous le titre : "Mécénat : l'entreprise partenaire ».

L'évolution du mécénat des entreprises met à jour les réactions à la crise: une mobilisation individuelle ou associée à des structures associatives qui mettent en place des projets à petite échelle mais qui répondent à des besoins locaux bien identifiés et dont on peut suivre l'évolution. Responsabilisation de l'individu-salarié dans l'entreprise mécène où il est en même temps le "parrain ", porteur du projet qu'il a souvent identifié et défendu auprès de l'entreprise. Dans tous les cas, (Fondation Vivendi, Fondation des Brasseries Kronenbourg, AxaAtout cœur, Fondation Rhône-Poulenc-Institut de France...) 
il n'est presque plus d'actions de mécénat sans un parrain-salarié dans l'entreprise mécène.

Il s'agit là d'un mécénat d'initiative, certes, mais l'initiative est de plus en plus souvent celle du salarié dont l'attention aux besoins qu'il perçoit dans son environnement est vivement appréciée par l'entreprise.

\section{Un mécénat solidaire}

78 Le salarié fait le lien, par sa démarche individuelle de solidarité, entre l'entreprise et la société de crise. C'est beaucoup moins l'entreprise qui engage sa responsabilité et ses fonds en matière de mécénat. Elle s'en remet aux choix et aux initiatives de ses salariés pour remédier aux effets de la crise dans la société. Elle propose, certes, une aide financière mais la formule de plus en plus fréquemment retenue est celle de doubler la mise du salarié.

Elf Aquitaine avait initié cette formule de "mécénat associé » dans laquelle l'entreprise s'engageait à doubler, sur un projet précis de mécénat, l'engagement financier des salariés. Plus novateur encore dans cet esprit, est le concept qui préside au fonctionnement de la Fondation Agir Pour l'emploi dont l'existence dépend totalement du soutien financier des salariés, chaque année à reconduire. La FAPE a été crée le 6 février 1995 par la volonté commune d'EDF, de Gaz de France, de la CFDT, de la CFECGT et de la CFTC. Les fonds de la FAPE proviennent des dons des salariés et d'une contribution équivalente des deux entreprises. Elle est complétée par un versement proportionnel au placement des salariés sur un plan d'épargne d'entreprise dédié au développement d'emplois dans les PME. Notons cependant que la provenance de ses fonds ainsi que sa gestion paritaire avec trois fédérations syndicales en font une fondation aujourd'hui unique en France. À ce titre, elle n'est pas une fondation d'entreprise au sens de la loi du 4 juillet 1990. Elle est abritée par la Fondation de France.

Cette interdépendance manifeste, constitutive de son fonctionnement, et le rôle essentiel de l'engagement financier des salariés, trouvent un écho dans les critères qui président au choix des champs d'intervention de la FAPE, notamment: «Être accessible au plus grand nombre », c'est-à-dire que "dans son appui aux services de proximité, le comité exécutif veille à ce que ces services permettent d'améliorer le quotidien du plus grand nombre, pour éviter une dérive vers une société duale où seules les populations solvables auraient accès aux services. Il est donc important que les porteurs de tels projets cherchent à obtenir la participation des mutuelles ou collectivités locales, notamment lors des phases de lancement de ces services ». Autre critère essentiel pour la FAPE d'un mécénat solidaire : "Contribuer à reconstruire ou à entretenir de la cohésion sociale en responsabilisant les acteurs de terrain », ce qui signifie que «l'implication des différents acteurs (élus, habitants, associations, etc.) est un facteur essentiel de la réussite du projet. Elle conditionne souvent sa pérennité et la qualité des emplois créés. Cette implication est particulièrement sensible lorsqu'il s'agit d'un service de proximité ou d'une Régie de Quartier».

\section{Un mécénat de compétences}

81 Si l'entreprise mécène aligne maintenant le plus souvent son engagement financier sur celui de ses salariés, assurée ainsi de leur soutien, elle encourage par ailleurs le bénévolat. 
Certes, ce ne sont pas les mêmes entreprises, et l'on perçoit facilement la différence entre « la culture » d'une entreprise de Service Public comme EDF, Gaz de France et la culture d'un groupe privé comme AXA. On prendra en effet la mesure de l'évolution du mécénat d'entreprise vers le développement du bénévolat des salariés avec l'exemple d'Axa Atout Cœur, structure de mécénat pionnière dans ce domaine, et qui, le plus souvent, n'engage pas les fonds de l'entreprise mais incite vivement ses salariés au bénévolat. Son slogan est clair: "Ce que les Hommes d'AXA-UAP peuvent donner de plus précieux, c'est euxmêmes ». Une participation financière de l'entreprise existe ponctuellement mais elle est de plus en plus rare.

Par ailleurs, sur le modèle anglo-saxon, l'entreprise propose aussi, plutôt que de l'argent, le détachement de ses salariés dans les associations qui participent à la lutte contre l'exclusion. Cette réserve quant à l'engagement financier est aussi une prudence à l'égard des éventuelles restrictions budgétaires ou des licenciements qu'impose la rentabilité de l'entreprise. De plus, l'entreprise trouve ici un moyen de valoriser autrement que financièrement le savoir que le salarié a "capitalisé » dans l'entreprise et dont il réalise l'utilité sociale.

83 C'est ainsi que se développe un mécénat dit "de compétences »: ouverture de l'entreprise sur son environnement à partir duquel s'évalue cette compétence, attachement au travail bien fait dont profite l'entreprise mais aussi la collectivité. Par ailleurs, directement confrontés à la crise, les salariés se perçoivent comme des privilégiés moins enclin à revendiquer dans l'entreprise.

84 Le mécénat de crise évolue donc vers un mécénat volontariste qui engage individuellement chaque salarié : détaché de l'entreprise (formule de l'entreprise Marks \& Spencer) ou bénévole sur son temps libre (formule d'« Axa à tout cœur»), il se fait l'émissaire de l'entreprise dans une société où chacun doit se remettre en question pour conserver sa place.

L'entreprise, par une démarche nouvelle de mécénat véritablement solidaire et non plus " de solidarité ", travaille à sa propre insertion dans le tissu social. En individualisant ses salariés par leur choix personnel d'engagement, elle les responsabilise et les implique directement dans l'insertion sociale à laquelle elle aspire, recréant ainsi, en interne comme en externe, un lien social.

Elle a compris que pour maitriser les antagonismes latents prêts à surgir en période de crise, elle devait intégrer les interrogations parfois violentes de la société dans laquelle et par laquelle elle vit. Consciente de l'interdépendance sociale, au moins sur notre terrain d'observation, le mécénat, l'entreprise n'est plus « citoyenne » face à la société mais dans et par la société. Elle s'intègre et intègre. Elle est en effet contrainte à une «solidarité agissante ».

87 La manifestation de l'interdépendance sociale laisse-t-elle une place au mécénat lorsque, notamment l'Association internationale pour le partenariat ONG-entreprises (AIPEO) déclare lors de sa journée d'études du 16 mai 1998: «Il faut passer d'une solidarité charitable à une solidarité juridiquement reconnue entre partenaires égaux et responsables. Le développement n'est pas l'apanage des administrations ni des ONG. Le monde est «condamné » à une solidarité agissante »? Le mécénat de crise engage au moins une redéfinition par l'entreprise de la relation mécène. 


\section{L'entreprise contrainte à une " solidarité agissante »}

88 Lorsque les entreprises "plaident pour une "éthique " compatible avec le profit", expliquent à leurs actionnaires préoccupés des bénéfices, que cette évolution, perçue le plus souvent comme coûteuse, doit être favorisée dans l'intérêt d'une politique de développement durable (« sustainability » en anglais) que l'on peut définir comme « une approche qui répond aux besoins du présent sans mettre en péril la capacité des générations futures à satisfaire leurs propres besoins $»^{27}$ peut-on les désigner encore comme des entreprises mécènes?

Il faut souligner que ce récent souci des entreprises d'intégrer l'éthique dans l'économique n'est pas spontané. On retiendra l'exemple de Shell qui s'est montré précurseur en ce domaine après avoir pris conscience de la dégradation de son image. En 1995, le groupe anglo-néerlandais est attaqué par les organisations de défense des droits de l'homme « après l'exécution par les pouvoirs publics nigérians de neuf opposants de la communauté ogonie, dans le delta du Niger, hostiles à l'exploitation de leur territoire par le pétrolier ${ }^{28}$. En mars 1997, le groupe élabore une charte de "bonne conduite » qui explicite sa responsabilité, non seulement envers les actionnaires, les clients et le personnel mais aussi envers les sous-traitants et la collectivité. Le groupe annonce avoir réduit de $5 \%$ ses émissions de gaz à effet de serre par rapport au niveau de 90 et participe à la recherche pour le développement des énergies renouvelables comme le vent, le solaire et la biomasse.

Crise du mécénat, certes, émergence d'un mécénat de crise, sans aucun doute dans des nouvelles formes d'engagements solidaires pris par les salariés et soutenus par les entreprises. De la culture au culturel, du culturel au social, la crise a déplacé les terrains d'application mais le concept de mécénat d'entreprise garde sa cohérence.

91 Cependant, lorsque l'entreprise est contrainte à évoluer, à mettre sur le même plan dans son rapport annuel ses performances commerciales et ses engagements éthiques, comme c'est le cas d'une manière particulièrement visible chez Shell depuis deux ans, on peut s'en réjouir mais aussi s'interroger sur ce qui peut encore relever d'un mécénat d'entreprise, concept qui implique que l'entreprise n'obéisse pas à une contrainte intégrée mais s'engage par un choix libre même s'il ne peut être totalement désintéressé.

Le CFIE (Centre Français d'Information sur les Entreprises, association loi 1901) a un objectif de responsabilisation du citoyen-consommateur par l'information. Dans son journal, Impact Entreprises, il fournit des informations et des analyses nécessaires « si nous voulons faire des choix dans nos manières de consommer ou d'épargner et aussi faire progresser les valeurs sociales et environnementales au sein des entreprises $»^{29}[\ldots]$. L'objectif de cette Lettre est double: "inciter la société civile à s'impliquer, en connaissance de cause, dans les affaires des entreprises et convaincre les décideurs économiques d'être à l'écoute de l'opinion $»^{30}$.

Pourra-t-on encore, lorsque l'entreprise face à un consommateur-citoyen exigeant, sera commercialement contrainte à des engagements, certes similaires à ceux dont se flatte l'entreprise mécène, parler encore de mécénat? Les stratégies d'une entreprise contrainte, "instituée », peuvent-elles être encore apparentées à celles, théoriquement libres et en ce sens hautement honorables de l'entreprise mécène? 


\section{BIBLIOGRAPHIE}

BOUGNOUX Daniel, LE MOIGNE Jean-Louis, PRoulX Serge (sous la direction de), Arguments pour une méthode (autour d'Édgar Morin), colloque de Cerisy, Seuil, Paris, Mai 1990.

BACKMANN René \& BRAUMAN Rony, Les médias et l'humanitaire, CFPJ, Paris, 1996.

BOURDIEU Pierre, Le sens pratique, éd. de Minuit, Paris, 1980.

BOURDIEU Pierre, Raisons pratiques. Sur la théorie de l'action, Seuil, Paris, 1994.

BRAUMAN Rony, Le crime humanitaire, Somalie, Arléa, Paris, 1993.

CLAVERo Bartolomé, La grâce du don, Albin Michel, coll. L'évolution de l'humanité, Paris, 1996.

colombat Catherine, Le guide du Mécénat solidarité, Dunod, Paris, 1994.

DEBRAY Régis, L'État séducteur, Gallimard, Paris, 1993.

FERRY Luc, L'homme Dieu ou le sens de la vie, Grasset, Paris, 1996.

FOURNIER Ariane, Pratique du mécénat humanitaire et social - de la BA à la communication partage,

Eyrolles, Paris, 1993.

Le Culte de l'entreprise, Revue Autrement nº 100, Sept. 1988.

LEVINAS Emmanuel, Entre nous. Essai sur le penser à l'autre, Grasset, Paris, 1991.

LEVY André, « Us et abus de la notion de crise », in Revue Internationale de Psychosociologie, 1998, vol. IV, n 9, pp. 169-178.

LIPOVETSKY Gilles, Le crépuscule du devoir, Gallimard, Paris, 1992.

MAUSS Marcel, « Essai sur le don » in Sociologie et anthropologie, Coll. « Quadrige », Paris, 1950.

MENDEL Gérard, « Peut-on répondre à la « crise » sans modifier la structure des organisations ? ", in Revue Internationale de Psychosociologie, $\mathrm{n}^{\circ}$ 8, Printemps 98, pp. 39-44.

MORIN Édgar, « Pour une crisologie », La notion de crise, Communications n² 25, Seuil, Paris, 1976.

MORIN Édgar, L'Esprit du temps, Nécrose, Grasset, Paris, 1983. Rufin Jean-Christophe, Le Piège

humanitaire, Hachette, coll. « Pluriel », Paris, 1989.

RUFIN Jean-Christophe, L'aventure humanitaire, Gallimard, Paris, 1994.

SCHNEIDER Michel, La Comédie de la Culture, Seuil, Paris, 1993.

VAN DER ELST Nicole, « De la génération communication à la génération médiation », in Humanisme et entreprise $\mathrm{n}^{\circ} 231$, Octobre 98, pp. 85-99.

VESCIA Remo, Aujourd'hui le mécénat, Éditions Cercle d'Art, Coll. Diagonales, Paris, 1996.

VESCIA Remo, Le Mécénat, art de la communication, communication de l'art, Economica, Paris, 1987.

WALTER Jacques, « l'Entreprise saisie par l'humanitaire. Contribution à l'étude de la

reconnaissance d'un monde professionnel », in Recherches en Communication, 6, pp. 103-124.

WALTER Jacques (sous la direction de), Le Téléthon, l'Harmattan, Paris, 1998. 


\section{NOTES}

1. Morin Édgar, «Pour une crisologie », Communications, La notion de crise, $\mathrm{n}^{\circ} 25$, Seuil, 1976,

p. 149.

2. Ibid.

3. Morin Édgar, Ibid., p. 163.

4. D'Almeida Nicole, «De l'organisation artefact à l'organisation institution : la place de la communication », communication aux Journées d'Études du groupe Org \& Co, Aix-en-Provence, 3, 4 et 5 juin 1999.

5. D'Almeida Nicole, «De l'organisation artefact à l'organisation institution: la place de la communication ", communication aux Journées d'Études du groupe Org \& Co, Aix-en-Provence, 3, 4 et 5 juin 1999.

6. Bourdieu Pierre, Raisons pratiques, Seuil, 1996, p. 162.

7. Bourdieu Pierre, Raisons pratiques, Seuil, 1996, p. 162.

8. On peut se reporter entre autres aux travaux d'Axel Gryspeerdt, "Vous êtes des nôtres: tentatives d'élargissement de la sphère interne de l'entreprise par la communication et les comportements de relations publiques ", Communication et organisation, $\mathrm{n}^{\circ} 4$, novembre 1993, et d'Yves Winkin, «Les relations publiques : rituels du pouvoir et pouvoirs du rituel », Lettre Scienceculture, $\mathrm{n}^{\circ}$ 40, Paris, GRIT, mars-avril 1989.

9. Ibid.

10. Le Culte de l'entreprise, Entretien avec Claude Marti, Autrement, $\mathrm{n}^{\circ} 100$, septembre 1988, p. 29.

11. Schneider Michel, La Comédie de la culture, Seuil, 1993, pp. 178-179.

12. Morin Édgar, «Pour une crisologie ", La notion de crise, Communications, Seuil, 1976, $n^{\circ} 25$, p. 152.

13. Ibid.

14. Bourdieu Pierre, Opus. cit., p. 150.

15. Ibid.

16. «Ne faites pas semblant de ne pas reconnaitre cette personne qui ne fait rien pour la Bosnie » disait une affiche-miroir apposée en 1993 sur les murs de Paris. Cité par Rony Brauman dans Les médias et l'humanitaire, p. 57.

17. Rony Brauman, René Backmann, Les médias et l'humanitaire, éditions du CFPJ, Paris, 1996, p. 76.

18. «Un rapport de la Commission européenne révèle l'absence de contrôle des dépenses humanitaires ", Le Monde, mardi 6 octobre 1998, p. 34.

19. R. Brauman, op. cit., p. 40.

20. Lipovetski Gilles, Le crépuscule du devoir. L'éthique indolore des nouveaux temps démocratiques, Gallimard, coll « NRF Essais », 1992, cité dans Les médias et l'humanitaire par Rony Brauman, p. 160.

21. Morir. Édgar, « Pour une crisologie », op. cit., p. 152.

22. Schneider Michel, La Comédie de la culture, Seuil, 1993, p. 109.

23. Ibid., p. 98.

24. «Le mécénat en chiffres », La Lettre du mécénat, août-septembre 92, p. 8.

25. Ibid.

26. «Trois millions d'exclus », La Lettre du mécénat, février-mars 93, dossier spécial, p. 2.

27. Définition proposée par les dirigeants de Shell, reprise dans «Shell s'engage contre la corruption et pour les énergies propres », Dominique Gallois, Le Monde, mardi 27 avril 1999.

28. Ibid.

29. Lettre du 10 décembre 98 de Martial Cozette, Directeur du CFIE, aux abonnés d'Impact Entreprises. 


\section{RÉSUMÉS}

Considérant la politique de communication des entreprises par le mécénat, il conviendra de spécifier de quelle crise nous entendons observer les effets : crise sociale et économique des années 90 ou crise interne à l'entreprise?

Nous pourrons ainsi mettre en évidence la vulnérabilité du mécénat d'entreprise en ce qu'il reste le fait du « Prince », tant qu'il est au pouvoir, mais aussi l'adaptabilité nécessaire et fructueuse de cette forme de communication, par essence tournée vers l'intérêt de l'Autre, donc à l'écoute des mutations sociales dont elle harmonise l'intégration dans l'entreprise.

In considering the communication policies of private companies, as related to philanthropic giving, it is necessary to define what crisis we are referring to in order to observe its effects; do we mean the social and economic crisis of the 90 s in general, or a particular crisis related to individual companies? One could insist upon the vulnerability of philanthropie projects sponsored by private enterprise when they rely on the good will of the giver ; but one could, in a more positive light, insist upon the advantages of adapting a company's communication policy when it is focused on the interests of others and of the community. In this way, a firm's communication policy both reflects social changes as well as prepares the way for more harmonious but necessary adaptations within the firm.

\section{AUTEUR}

\section{NICOLE DENOIT}

L'auteur, Docteur de 3e cycle en Littérature française, enseigne à Tours, dans la filière «Civilisation - Communication » de l'UFR d'anglais à la Faculté des Lettres de l'Université François Rabelais. Elle s'intéresse actuellement à l'évolution du mécénat des entreprises des années 80 aux années 90 et prépare, sur ce sujet, une thèse à Bordeaux 3 en Sciences de l'Information et de la Communication. 